Ann. Zoolech., I065, 14 (3), 279-283.

\title{
IOTE SUR L'HÉRITABILITÉ DU RENDEMENT A L'ABATTAGE CHEZ LE POULET
}

\author{
F. H. RICARD \\ avec la collaboration technique de (i. MARChF et J. Hens. \\ Station expérimentale d'Aviculture du Magneraud, \\ Saint-Pierre-d'Amilly (Charente-Maritime)
}

SOMMAIRE:

Nous avons calculé le rendement à l'abattage (poids effilé/poids vif) pour 3 groupes de coquelets d'une même souche dite Bresse pile. Les 2 premiers groupes comprenaient chacun 145 animaux âgés de 87 jours, le troisième 316 animaux âgés de 77 jours.

Le rendement exprimé en pour cent du poids vif présente un faible coefficient de variation, de l'ordre de 1,5 p. Ioo. Mais la variabilité génétique n'est pas négligeable : pour les mesures brutes, les valeurs de l'héritabilité moyenne sont respectivement de $0,17,0,44$ et 0,40 avec un écart-type correspondant de $0,14,0,22$ et 0,17 . Les mesures transformées en probits conduisent à des héritabilités légèrement supérieures.

\section{INTRODUCTION}

De nombreux facteurs peuvent influencer le rendement à l'abattage du poulet : l'âge, le sexe, la souche, l'alimentation, les conditions d'élevage et d'attrapage des animaux. Quand tous ces facteurs sont maintenus constants, il reste une variabilité due aux différences génétiques entre familles et aux facteurs aléatoires de l'environnement. L'étude de cette variabilité permet d'estimer l'héritabilité et de voir dans quelle mesure on peut améliorer le rendement à l'abattage par la sélection.

Analysant une série de croisements diallèles, Murr et GoOdman (I964) ont trouvé une héritabilité due à l'action additive des gènes faible $\left(0, I_{4}\right)$ par rapport à l'héritabilité non additive $(0,37)$. L'analyse de poulets abattus au Magneraud à l'occasion de différentes expériences, nous permet d'apporter une contribution à la connaissance de la variabilité génétique du rendement à l'abattage. 


\section{MATÉRIEL ET TECHNIQUES}

Les animaux étudiés étaient des coquelets d'une souche expérimentale, dite Bresse Pile, pris dans la descendance de ro coqs du cheptel ig6o et de 8 coqs du cheptel I962, chaque coq étant accouplé à 4 poules.

Pour le prélèvement de 1960,3 des 4 poules accouplées au même coq ont fourni 8 descendants et la quatrième 5, soit un total de 290 animaux, répartis en 8 lots d'éclosion. La souche était divisée en 2 lignées élevées ensemble : la moitié des animaux appartenait à la lignée $N$, issue de croisements New Hampshire $\times$ Bresse blanche effectués en I955 et sélectionnés depuis cette date en troupeau fermé ; l'autre lignée, dite $W$, correspondait à des animaux $Y$ croisés avec des White American Pour le prélèvement I 962 , nous avions prévu ro descendants par couple coq-poule. In réalité une poule sur les 32 n'a pu en donner que 8 et 2 en ont donné 9, soit un total de 316 animaux répartis en ro lots d'éclosion. Tous les animaux de 1962 appartenaient à la lignée $N$, maintenue sans sélection depuis 1960 .

L'alimentation était constituée par une pâtée sèche distribuée à volonté. Le tableau I en donne les caractéristiques principales. Fin I 960, il s'agissait d'un aliment "croissance " classique comprenant la pâtée $A_{1}$ donnée de l'éclosion à 3 semaines puis la pâtée $A_{2}$ donnée de 3 seinaines à l'abattage. En 1962, les animaux ont reçu le seul aliment B, de type " engraissement ", de l'éclosion à l'abattage.

\section{TABI,EAU I}

\section{Caractíristiques générales des aliments uitisés}

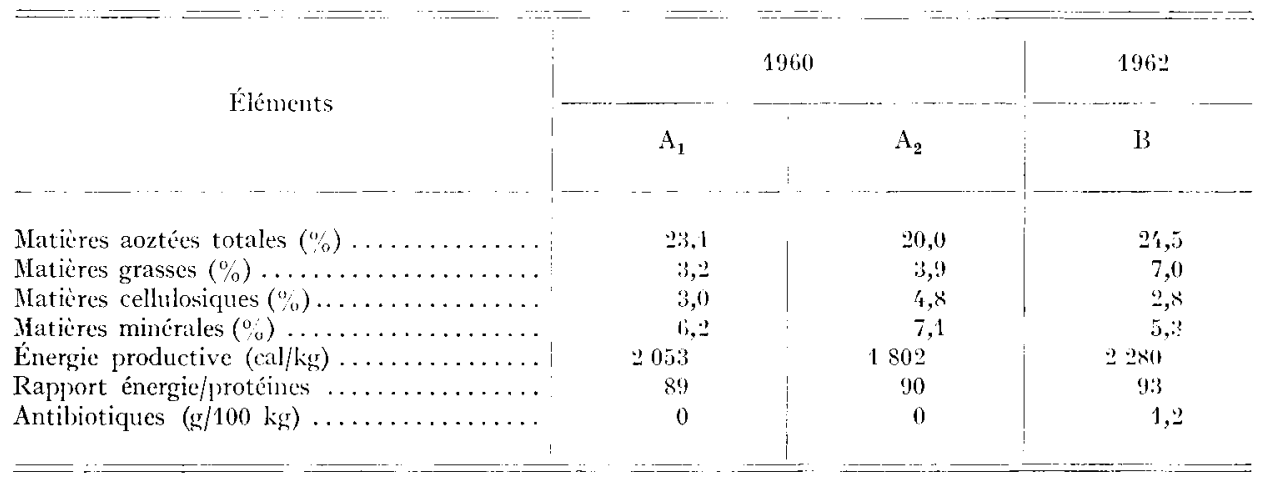

Les poulets ont été abattus après un jeûne complet d'environ 15 heures, à l'âge de 87 jours pour la série 1960 et de 77 jours pour la série I 962 . Ils étaient saignés par section des vaisseaux au fond de la gorge, échaudés, plumés à l'aide d'une machine à doigts de caoutchouc avec finition à la main, puis effilés. Cette dernière opération consiste à enlever de la carcasse plumée l'ensemble intestins + pancréas.

Le poids vif était noté immédiatement avant l'abattage et le poids de la carcasse effilée après 8 heures de ressuyage à la température du laboratoire. Le rendement à l'abattage est défini par :

$$
x=\frac{\text { poids carcasse effilée } \times 100}{\text { poids vif avant abattage }}
$$

Nous avons calculé les paramètres statistiques et les composantes de l'héritabilité de la variable $x$ pour les 2 groupes de poulets 1960 , l'ensemble des 2 groupes 1960 considérés comme ne faisant qu'une seule souche, et le groupe des poulets 1962. Les calculs de base ont été réalisés dans l'atelier de mécanographie du Magneraud.

Le nombre de poules par coq étant le même et le nombre d'enfants par poule peu différent, nous avons pu estimer les héritabilités et leurs écarts-types selon la technique simplifiée décrite 
par Graybill et Robertson (1957). Nous avons considéré que chaque poule avait donné une moyenne de 7,25 descendants en 1960 et 9,875 en 1962. D'ailleurs, le calcul direct par la méthode de KING et HENDERSON (I954), utilisée sans tenir compte du lot d'éclosion, a abouti à une différence de 0 ,or dans 2 cas et à un résultat identique dans tous les autres cas.

Pour éliminer une influence possible du lot d'éclosion, nous avons refait les calculs d'héritabilité sur les probits de valeurs brutes, calculés à partir des histogrammes de fréquences cumulées selon le procédé de Cochez et Pero (I954). Nous avons obtenu les fréquences en divisant l'effectif de chaque classe de l'histogramme par $\mathrm{N}+$ I (nombre total d'animaux du lot plus un), ce qui permet de régulariser la valeur des probits aux 2 extrémités des histogrammes quand l'effectif total est faible.

\section{RÉSULTATS ET DISCUSSION}

Dans le tableau 2, notıs avons résumé les caractéristiques de chaque groupe d'animaux (nombre, âge, poids vif moyen) et les paramètres statistiques du rendement à 1'abattage.

TABLEAU 2

Caractéristiques des animaux et paramètres statistiques du rendement à l'abattage

\begin{tabular}{l|c|c|c|c|c|c}
\hline \hline \multicolumn{1}{c}{ Animaux } & \multicolumn{2}{c}{ Rendement à l'abattage } \\
\hline Groupe & Effectif & $\begin{array}{c}\text { Age } \\
\text { en jours }\end{array}$ & $\begin{array}{c}\text { Poids vif } \\
\text { en grammes }\end{array}$ & $\begin{array}{c}\text { Moyenne } \\
(\%)\end{array}$ & $\begin{array}{c}\text { Écart-type } \\
(\%)\end{array}$ & $\begin{array}{c}\text { Coefficient } \\
\text { variation (\%) }\end{array}$ \\
\hline 1960 lignée $N$ & 145 & 87 & 1742 & 80,87 & 1,27 & $\mathbf{1 , 5 7}$ \\
\hline $\mathbf{1 9 6 0}$ lignée $W$ & 145 & 87 & 1839 & 80,95 & 1,53 & 1,89 \\
\hline Ensemble 1960 & 290 & 87 & 1791 & 80,91 & 1,41 & 1,74 \\
\hline Ensemble 1962 & 316 & 77 & 1477 & 81,35 & 1,15 & 1,41 \\
\hline \hline
\end{tabular}

Parmi les animaux de I960, ceux de la lignée $W$ sont plus lourds que ceux de la lignée $N$ mais les rendements à l'abattage ne sont pas significativement différents $(t=0,48)$. Il semble donc logique de regrouper les 2 lignées ce qui permet d'augmenter le nombre de degrés de liberté dans les calculs d'héritabilité. Au contraire, le groupe Ig62 présente un rendement à l'abattage significativement plus élevé que le groupe Ig60 $(t=4, \mathrm{Ig})$. Les changements survenus entre les 2 prélèvements peuvent expliquer cette différence, en particulier 1'aliment qui était nettement plus riche en I962. Or, HARMs et al. (I957) ont obtenu une augmentation du rendement à 1'abattage en augmentant le rapport énergie/protéine de la ration.

Les coefficients de variation intra-groupe sont faibles, de l'ordre de r,5 p. Ioo. Il semble que ce fait résulte principalement du mode de calcul du rendement, exprimé en pourcentage. Notons que pour les centres d'abattage qui manipulent des tonnages importants, une différence de l'ordre de I p. Ioo peut représenter une somme d'argent considérable. 
Le tableau 3 donne pour chaque groupe les composantes mère et père de 1'héritabilité, la valeur moyenne ainsi que les écarts-types correspondants.

\section{TABLEAU 3}

Héritabilités du rendement à l'abattage et écarts-types correspondanls

\begin{tabular}{|c|c|c|c|c|c|c|}
\hline & \multicolumn{2}{|c|}{ Composante mère } & \multicolumn{2}{|c|}{ Composante père } & \multicolumn{2}{|c|}{ Moyenne } \\
\hline & $h_{m}^{2}$ & $s$ & $h_{\nu}^{2}$ & $s$ & $h_{\rho}^{2} n_{b}$ & s \\
\hline $1^{0}$ Valeurs brutes & & & & & & \\
\hline 1960 lignée $N \ldots \ldots$. & 0,26 & 0,28 & 0,08 & 0,20 & 0,17 & 0,14 \\
\hline 1960 lignée $W \quad \ldots \ldots$ & 0,38 & 0,29 & 0,50 & 0,45 & $0,4^{4} *$ & 0,22 \\
\hline Ensemble $1960 \ldots \ldots$. & $0,3 \mathbf{t}$ & 0,21 & 0,27 & 0,22 & $0,31 *$ & 0,12 \\
\hline Ensemble 1962 . & 0,18 & 0,15 & 0,62 & 0,34 & $0,10^{*}$ & 0,17 \\
\hline $2^{\mathrm{e}}$ Probits & & & & & & \\
\hline Ensemble 1960 & 0,41 & 0,22 & 0,33 & 0,25 & $0,37 * *$ & 0,14 \\
\hline Ensemble 1962 & 0,17 & 0,14 & 0,69 & 0,37 & $0,43^{*}$ & 0,18 \\
\hline
\end{tabular}

$(*)=$ héritabilité significativement différente de zéro au seuil 5 p. 100

$\left(*^{*}\right)=$ héritabilité significativement différente de zéro au seuil 1 p. 100

Nous n'observons pas de tendance générale quant à la valeur relative des composantes mère et père. La différence des formules alimentaires utilisées en I 960 et I 962 pourrait expliquer en partie ce résultat : Boyer et al. (I963) obtiennent des héritabilités plus fortes avec un aliment riche en énergie, le phénomène étant particulièrement net au niveau de la composante père. Dans notre cas, il faut également considérer que les effectifs sont faibles si bien que tous les intervalles de confiance comprennent la valeur zéro.

Les héritabilités moyennes observées sont significatives dans le cas de la lignée $W$, de l'ensemble I 960 et du groupe I $962: 30$ à $40 \mathrm{p}$. Ioo des différences entre individus sont dues à l'action additive des gènes. Murr et Goodman (Ig64) trouvent une valeur plus faible : o,I4. En plus du fait que la souche étudiée et les conditions d'élevage n'étaient pas les mêmes, la différence peut s'expliquer par les erreurs d'échantillonnage.

Dans le cas de nos animaux, le lot d'éclosion avait un effet significatif sur les moyennes : $\mathrm{F}=3,96$ pour l'ensemble des animaux I96o et $\mathrm{F}=8,82$ pour le groupe I962. La transformation " probit " permet théroriquement d'éliminer l'influence du milieu, en particulier du lot (Cochez et PERo, I954), et doit donc augmenter l'estimation de l'héritabilité. Dans le cas présent, les valeurs obtenues sont à peine supérieures à celles qui résultent du calcul sur valeurs brutes (cf. tabl. 3). On peut penser que ceci est dû à ce que les familles étaient bien représentées dans les différents lots d'éclosion.

En conclusion, nos données montrent que pour la souche étudiée, il existe des 
différences génétiques entre familles et que le rendement à l'abattage est un caractère sélectionnable. Des calculs portant sur des échantillons plus nombreux et sur d'autres souches permettraient de voir s'il est possible de généraliser nos résultats.

Reçu pour publication en juin 1965

\title{
SUMMARY
}

\author{
A NOTE ON DRESSING YIELD HERITABILITY IN CHICKENS
}

In 3 groups of chickens from the so-called Bresse pile strain, dressing yield was calculated as live weight percentage of the french dressed carcasses. Table 2 gives the number of chickens in each group, age and weight at slaughter, and statistical parameters of dressing yields; table 3 gives components and standard deviations of heritabilities.

Variations cofficients are low, about I.5 p. I00, but in our strain genetic variability does exist. The mean heritability values for the 3 groups are $0.17,0.44$, and 0.40 respectively with standard deviations of $0.14,0.22$, and 0.17 . A pseudo-probit transformation of the data led to lightly greater values.

\section{RÉFÉRENCES BIBLIOGRAPHIQES}

Boyer J. P., de LAnge X., Calet C., I 963 . Influence of feed on the heritability of some chicken traits. Proc. 11 th. intern. Congr. Genet., The Hague. 1, 262 (Abstract).

Cochez L. P., Pero R., 1954. Sélection des caractères économiques. Transformation des mesures brutes en écarts réduits pour atténuer l'incidence des variations de milieu. Proc. 10 th World's Poult. Congr., Edinburg., I6-I9.

Graybill F. A., Robertson W. H., I957. Calculating confidence intervals for genetic heritability. Poult. Sci., 36, 26I-265.

Harms R. H., Hochreich H. J., Meyer B. H., 1957. The effect of feeding 3 energy levels upon dressing percentages and cooking losses of White Rock broiler-fryers, Poult. Sci., 36, 420-422.

King S. C. n Henderson C. R., 1954. Variance component analysis in heritability studies. Poult. Sci., 33, I47-I 54 .

MuIR F. V., Goodman B. I., I964. Heritability of dressing percentage in broilers. Poult. Sci., 43, I605-1606. 